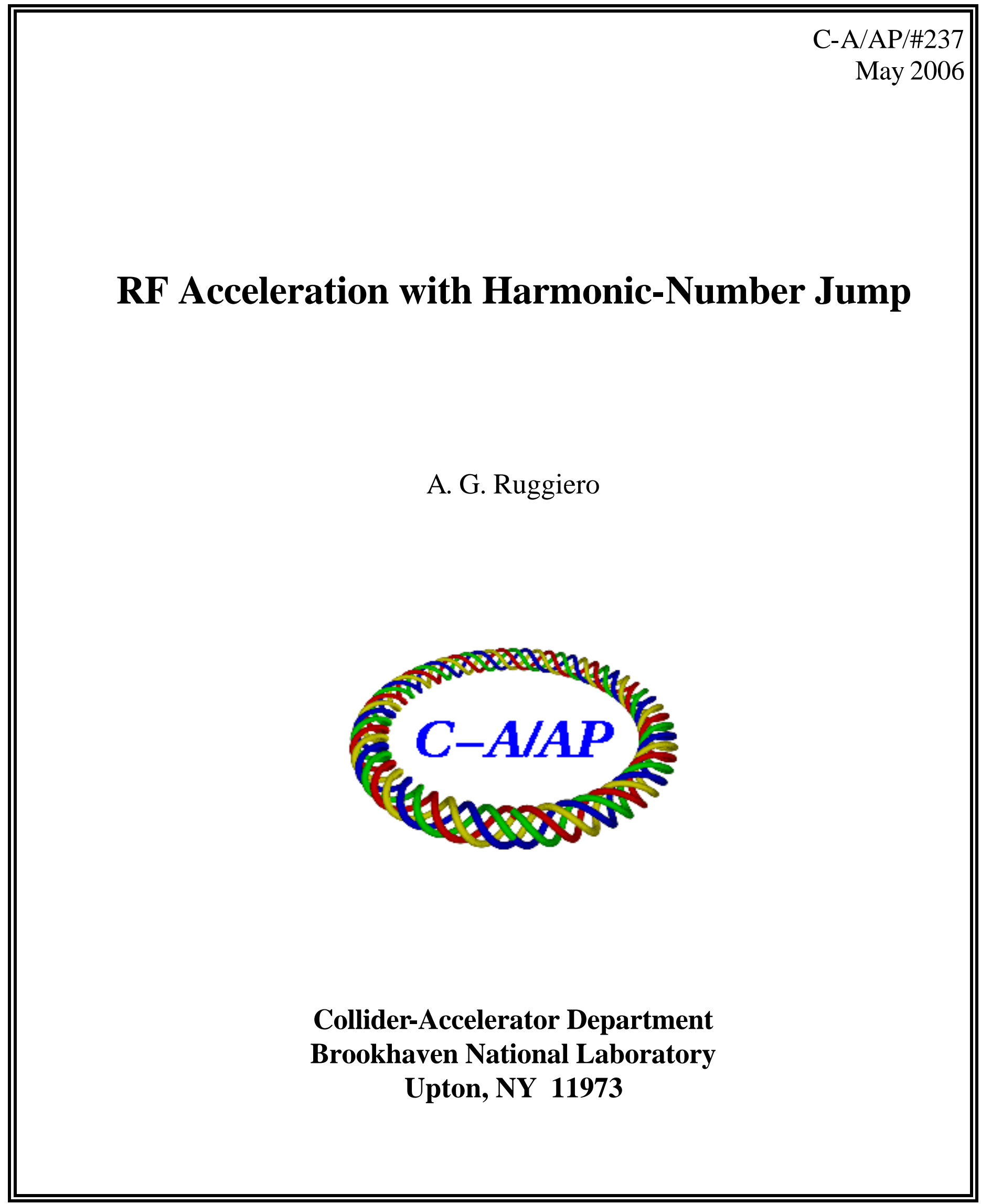




\title{
RF Acceleration with Harmonic-Number Jump
}

\author{
Alessandro G. Ruggiero \\ Brookhaven National Laboratory \\ May 10, 2006
}

\section{Abstract}

We have recently considered acceleration of protons and heavy ions in a FixedField Alternating-Gradient (FFAG) accelerator with Non-Scaling Lattice and Linear Field Profile [1]. To avoid the problem of frequency modulation for acceleration of lowenergy beams over a too short period of time, and to boost the acceleration rate, the method of Harmonic-Number Jump (HNJ) was proposed [2]. This method allows the use of constant frequency acceleration, for instance using superconducting cavities, despite the fact that the beam velocity may vary considerably. Of course in this case the accelerating voltage and RF phase will need to be programmed accordingly as we shall show. We shall study first the motion of Synchronous particles, and then of those with deviating initial conditions. We estimate the area and height of the RF buckets that are to contain the beam bunches with the added condition of the HNJ. We finally investigate methods to allow the program of energy gain as required by the HNJ method, including the effect of the cavity Transit Time Factor (TTF).

\section{Acceleration of Synchronous Particles}

Let us assume that the FFAG ring is made of N RF cavities equally spaced, and that the lattice properties and structure between two consecutive cavities repeat identically around the ring (see Figure 1). The acceleration cycle is then thought as a sequence of pair events: an energy kick at the location of the n-th cavity, followed by an $\operatorname{arc} A_{n}$ that takes the beam from that cavity to the next.

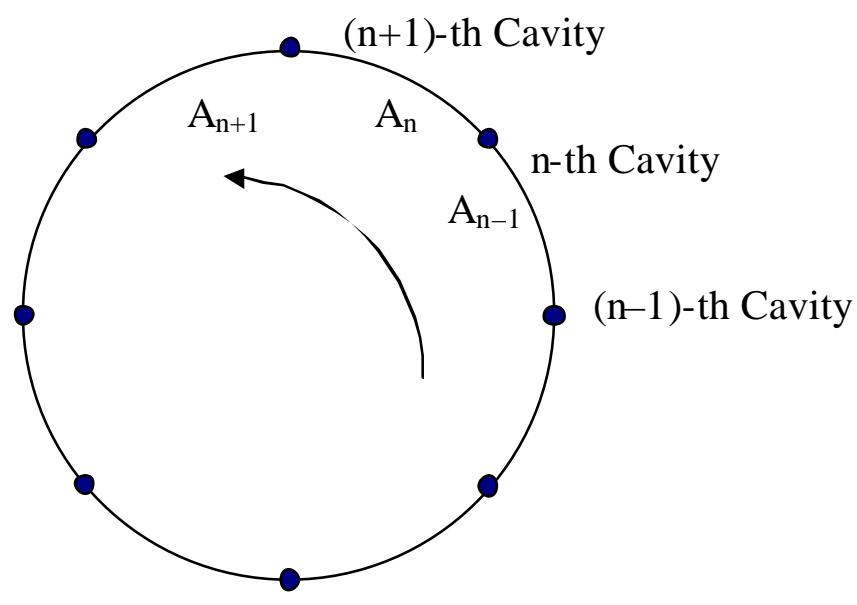

Figure 1. FFAG ring with multiple equally-spaced RF Cavities 
Let us start considering a beam made of point-like bunches; that is, all particles have the same energy, and cross the cavities all together at the same instant. Equivalently each point-like bunch can be though to be a synchronous or a reference particle around which all other particles oscillate. The total energy of the reference particle in the $\mathrm{n}$-th arc $A_{n}$ is $\mathbf{E}_{n}$, and the period of time that it takes to travel the arc $A_{n}$ is $\mathbf{T}_{n}=h_{n} T_{R F}$, where $T_{R F}$ is the constant RF period, and $h_{n}$ is the RF harmonic number local to the arc $A_{n}$. Here and in the following bold face parameters apply to the reference particle. The same parameters in plain face apply to the other particles.

Let $\mathrm{Q}$ and $\mathrm{A}$ denote respectively the charge state and the mass number of the ion particle. The energy gain when crossing the n-th cavity is

$$
\begin{aligned}
\Delta \mathbf{E}_{\mathrm{n}} & =\left(\mathrm{Q} e \mathrm{~V}_{\mathrm{n}} / \mathrm{A}\right) \sin \left(\omega_{\mathrm{RF}} \mathbf{t}_{\mathrm{n}}\right) \\
& =(\mathrm{Q} \mathrm{eV} / \mathrm{A}) \sin \left(\phi_{\mathrm{n}}\right)
\end{aligned}
$$

where $V_{n}$ is the peak voltage, $\omega_{R F} / 2 p$ the RF frequency, $t_{n}$ the instant of traversal of the cavity, and $\phi_{\mathrm{n}}=\omega_{\mathrm{RF}} \mathbf{t}_{\mathrm{n}}$ the RF synchronous phase. Both $\mathrm{V}_{\mathrm{n}}$ and $\phi_{\mathrm{n}}$ can vary from cavity to cavity, and, in the same cavity, from turn to turn.

The HNJ method requires that the energy gain is adjusted to cause a change in the travel period $\mathbf{T}_{\mathrm{n}}$ in the following arc $\mathrm{A}_{\mathrm{n}}$, so that the reference particle is pushed forward or back exactly by $\Delta \mathrm{h}$ RF harmonics and appears in an exactly identical bucket ahead or trailing by $\Delta \mathrm{h}$ RF wavelengths (see Figure 2). Thus, by denoting

$$
\begin{array}{lll}
\mathbf{T}_{\mathrm{n}} & =\mathrm{h}_{\mathrm{n}} \mathrm{T}_{\mathrm{RF}} \\
\mathbf{T}_{\mathrm{n}-1}= & \mathrm{h}_{\mathrm{n}-1} \mathrm{~T}_{\mathrm{RF}} \\
\mathrm{h}_{\mathrm{n}}- & \mathrm{h}_{\mathrm{n}-1}=-\Delta \mathrm{h}
\end{array}
$$

this is accomplished by requiring, in linear approximation,

$$
\Delta \mathbf{E}_{\mathrm{n}}=\beta_{\mathrm{n}}^{2} \gamma_{\mathrm{n}}^{3} \mathrm{E}_{0} \Delta \mathrm{h} / \mathrm{h}_{\mathrm{n}}\left(1-\alpha_{\mathrm{pn}} \gamma_{\mathrm{n}}^{2}\right)
$$

where $E_{0}$ is the ion particle rest energy, $\beta_{\mathrm{n}}$ and $\gamma_{\mathrm{n}}$ the usual relativistic velocity and energy factors, and $\alpha_{\mathrm{pn}}$ the momentum compaction factor local to the $n$th $\operatorname{arc} A_{n}$. The HNJ is executed by combining Eq.s (1, 2 and 3).

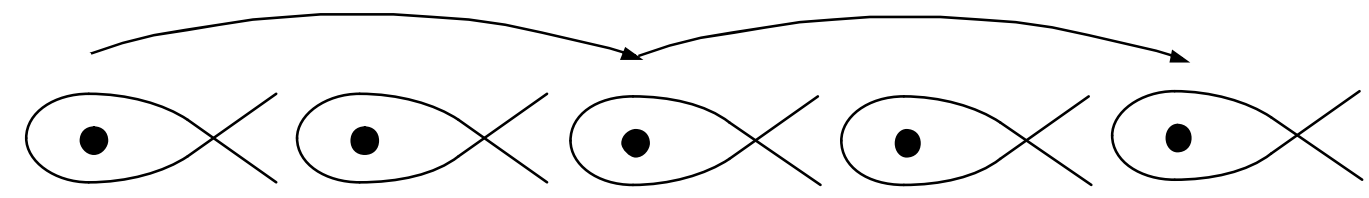

Figure 2. RF Bucket Jump at Cavity Crossings by $\Delta \mathrm{h}$ RF wavelengths (harmonic number) 


\section{Acceleration of Non-Synchronous Particles}

Let us consider now an off-momentum particle following (or heading) the reference particle with a time difference $\tau_{n}$. It will cross the $n$-th cavity at the instant $t_{n}=$ $\mathbf{t}_{\mathrm{n}}+\tau_{\mathrm{n}}$, and the corresponding energy gain is

$$
\Delta \mathrm{E}_{\mathrm{n}}=\left(\mathrm{Q} e \mathrm{~V}_{\mathrm{n}} / \mathrm{A}\right) \sin \left(\omega_{\mathrm{RF}} \mathrm{t}_{\mathrm{n}}\right)
$$

Subtracting side by side Eq. (1) from Eq. (4) gives the change in the energy difference $\varepsilon_{\mathrm{n}}=\mathrm{E}_{\mathrm{n}}-\mathbf{E}_{\mathrm{n}}$ after crossing the $\mathrm{n}$-th cavity

$\Delta \varepsilon_{\mathrm{n}}=\left(\mathrm{Q} e \mathrm{~V}_{\mathrm{n}} / \mathrm{A}\right)\left[\sin \left(\phi_{\mathrm{n}}+\omega_{\mathrm{RF}} \tau_{\mathrm{n}}\right)-\sin \left(\phi_{\mathrm{n}}\right)\right]$

In the limit of small delay $\tau_{\mathrm{n}}$

$$
\Delta \varepsilon_{\mathrm{n}} \quad=\quad\left(\mathrm{Q} e \mathrm{~V}_{\mathrm{n}} / \mathrm{A}\right)\left(\cos \phi_{\mathrm{n}}\right) \omega_{\mathrm{RF}} \tau_{\mathrm{n}}
$$

The change of the time delay, in linear approximation, is given by

$$
\begin{aligned}
\Delta \tau_{\mathrm{n}} & =\tau_{\mathrm{n}}-\tau_{\mathrm{n}-1} \\
& =\quad\left(\mathrm{t}_{\mathrm{n}}-\mathbf{t}_{\mathrm{n}}\right)-\left(\mathrm{t}_{\mathrm{n}-1}-\mathbf{t}_{\mathrm{n}-1}\right) \\
& =\mathrm{T}_{\mathrm{n}}-\mathbf{T}_{\mathrm{n}} \\
& =-\left(1-\alpha_{\mathrm{pn}} \gamma_{\mathrm{n}}^{2}\right) \mathbf{T}_{\mathrm{n}} \varepsilon_{\mathrm{n}} / \beta_{\mathrm{n}}{ }^{2} \gamma_{\mathrm{n}}^{3} \mathrm{E}_{0}
\end{aligned}
$$

Combining Eq.s (6 and7) gives the phase (or energy) oscillation equation in the limit of small amplitude

$$
\Delta^{2} \tau_{\mathrm{n}} / \Delta \mathrm{n}^{2} \quad+\quad \Omega_{\mathrm{n}}^{2} \tau_{\mathrm{n}}=0
$$

where the angular oscillation frequency

$$
\Omega_{\mathrm{n}}^{2}=2 \mathrm{p} \Delta \mathrm{h} / \operatorname{tg} \phi_{\mathrm{n}}
$$

\section{Motion within RF Buckets}

The two canonical Eq.s (5 and 7) can be derived from the following Hamiltonian

$$
\begin{aligned}
\mathrm{H} & =\left(\mathrm{Q} e \mathrm{~V}_{\mathrm{n}} / \mathrm{A} \omega_{\mathrm{RF}}\right)\left[\cos \left(\phi_{\mathrm{n}}+\omega_{\mathrm{RF}} \tau_{\mathrm{n}}\right)+\omega_{\mathrm{RF}} \tau_{\mathrm{n}} \sin \left(\phi_{\mathrm{n}}\right)\right]+ \\
& -\left(1-\alpha_{\mathrm{pn}} \gamma_{\mathrm{n}}{ }^{2}\right) \mathbf{T}_{\mathrm{n}} \varepsilon_{\mathrm{n}}{ }^{2} /\left(2 \beta_{\mathrm{n}}{ }^{2} \gamma_{\mathrm{n}}{ }^{3} \mathrm{E}_{0}\right)
\end{aligned}
$$

The motion of all particles is to occur in RF buckets as the one shown in Figure 3. 


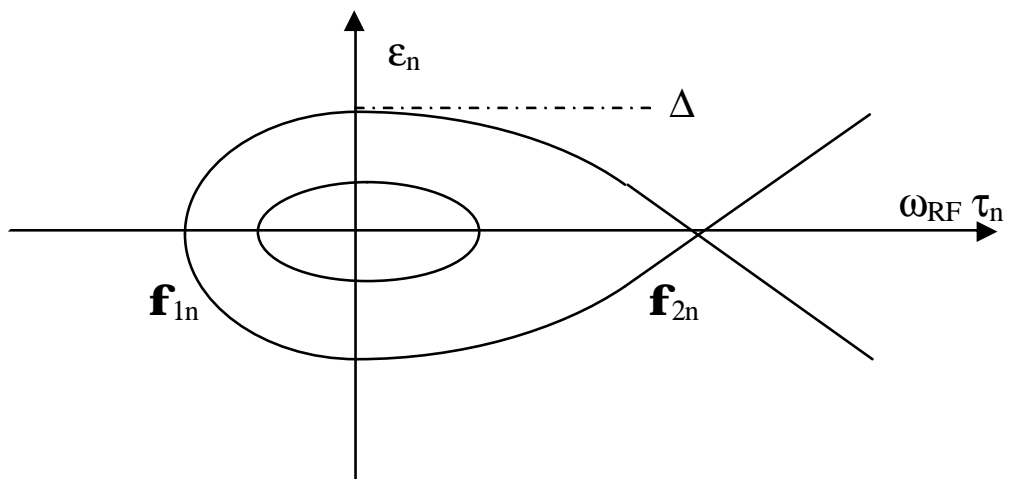

Figure 3. Separatrix of RF Bucket

From Eq. (5) we see that there are two fixed points at

$\omega_{\mathrm{RF}} \tau_{\mathrm{n}}=0 \quad$ (stable)

$\omega_{\mathrm{RF}} \tau_{\mathrm{n}}=\mathrm{p}-2 \phi_{\mathrm{n}} \quad=\quad \phi_{2 \mathrm{n}} \quad$ (unstable)

The value of the bucket separatrix is given by letting $\varepsilon_{\mathrm{n}}=0$ and $\omega_{\mathrm{RF}} \tau_{\mathrm{n}}=\mathrm{p}-2 \phi_{\mathrm{n}}$ in Eq. (10), that is

$\mathrm{H}_{\mathrm{n}} \quad=\quad-\left(\mathrm{Qe} \mathrm{V}_{\mathrm{n}} / \mathrm{A} \omega_{\mathrm{RF}}\right)\left[\cos \left(\phi_{\mathrm{n}}\right)-\left(\mathrm{p}-2 \phi_{\mathrm{n}}\right) \sin \left(\phi_{\mathrm{n}}\right)\right]$

The RF bucket extends over the phase range between $\omega_{\mathrm{RF}} \tau_{\mathrm{n}}=\phi_{1 \mathrm{n}}$ and $\omega_{\mathrm{RF}} \tau_{\mathrm{n}}=\phi_{2 \mathrm{n}}$, that are the roots of the following equation

$$
\cos \left(\phi_{\mathrm{n}}+\omega_{\mathrm{RF}} \tau_{\mathrm{n}}\right)+\cos \left(\phi_{\mathrm{n}}\right)+\left(\omega_{\mathrm{RF}} \tau_{\mathrm{n}}-\mathrm{p}+2 \phi_{\mathrm{n}}\right) \sin \left(\phi_{\mathrm{n}}\right)=0
$$

The two phase angles $\phi_{1 \mathrm{n}}$ and $\phi_{2 \mathrm{n}}$ are plotted versus $\phi_{\mathrm{n}}$ in Figure 4 .

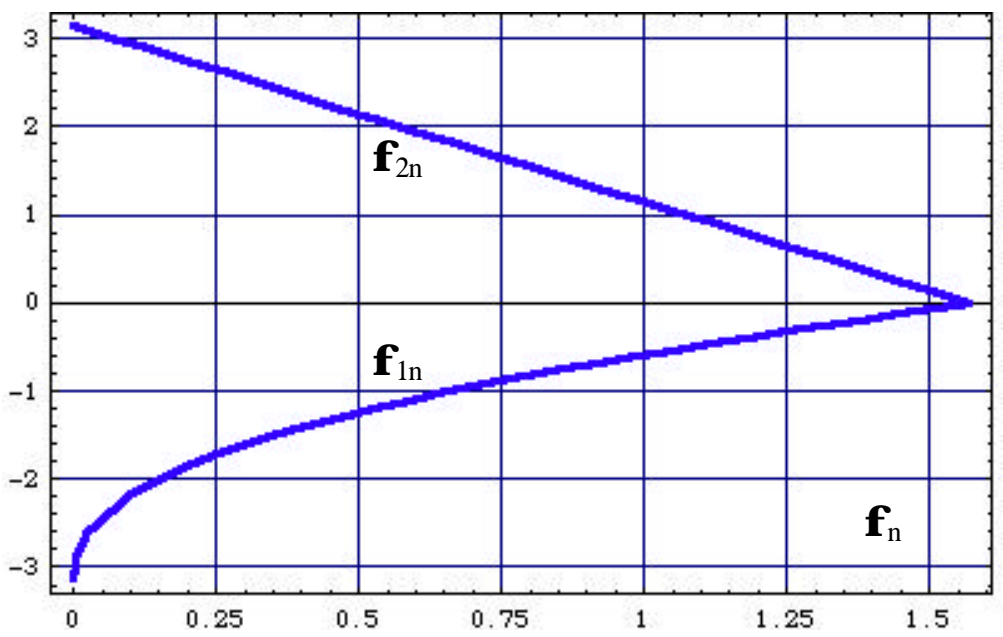

Figure 4. Plot of $\phi_{1 \mathrm{n}}$ and $\phi_{2 \mathrm{n}}$ versus $\phi_{\mathrm{n}}$ 
We can also find the bucket half height

$\Delta^{2}=2$ Qe $V_{n} \beta_{n}^{2} \gamma_{n}^{3} E_{0} F\left(\phi_{n}\right) / A \pi\left(1-\alpha_{p n} \gamma_{n}^{2}\right) h_{n}$

where

$\mathrm{F}\left(\phi_{\mathrm{n}}\right)=\cos \left(\phi_{\mathrm{n}}\right)-\left(\mathrm{p} / 2-\phi_{\mathrm{n}}\right) \sin \left(\phi_{\mathrm{n}}\right)$

which is plotted in Figure 5. Similarly we can calculate the area $B_{n}$ surrounded by the RF bucket. This can be expressed as

$\mathrm{B}_{\mathrm{n}}=\mathrm{B}_{0 \mathrm{n}} \mathrm{I}\left(\phi_{1 \mathrm{n}}, \phi_{2 \mathrm{n}}\right)$

with

$\mathrm{B}_{0 \mathrm{n}}=\left(8 / \omega_{\mathrm{RF}}\right)\left[2 \mathrm{Qe} \mathrm{V}_{\mathrm{n}} \beta_{\mathrm{n}}{ }^{2} \gamma_{\mathrm{n}}{ }^{3} \mathrm{E}_{0} / \mathrm{A} \pi \mathrm{h}_{\mathrm{n}}\left(1-\alpha_{\mathrm{pn}} \gamma_{\mathrm{n}}{ }^{2}\right)\right]^{1 / 2}$

and

$\mathrm{I}\left(\phi_{1 \mathrm{n}}, \phi_{2 \mathrm{n}}\right)=\int_{\phi_{1 \mathrm{n}}}^{\phi_{2 \mathrm{n}}}\left[\cos \left(\phi_{\mathrm{n}}+\phi\right)+\phi \sin \left(\phi_{\mathrm{n}}\right)+\mathrm{G}\left(\phi_{\mathrm{n}}\right)\right]^{1 / 2} / 4 \sqrt{2} \mathrm{~d} \phi$

where

$\mathrm{G}\left(\phi_{\mathrm{n}}\right)=\cos \left(\phi_{\mathrm{n}}\right)-\left(\mathrm{p}-2 \phi_{\mathrm{n}}\right) \sin \left(\phi_{\mathrm{n}}\right)$

This integral is plotted in Figure 6 versus the RF phase . As a curiosity observe that the two functions $\mathrm{I}\left(\phi_{1 \mathrm{n}}, \phi_{2 \mathrm{n}}\right)$ and $\mathrm{F}\left(\phi_{\mathrm{n}}\right)$ are almost equal within a few percent.

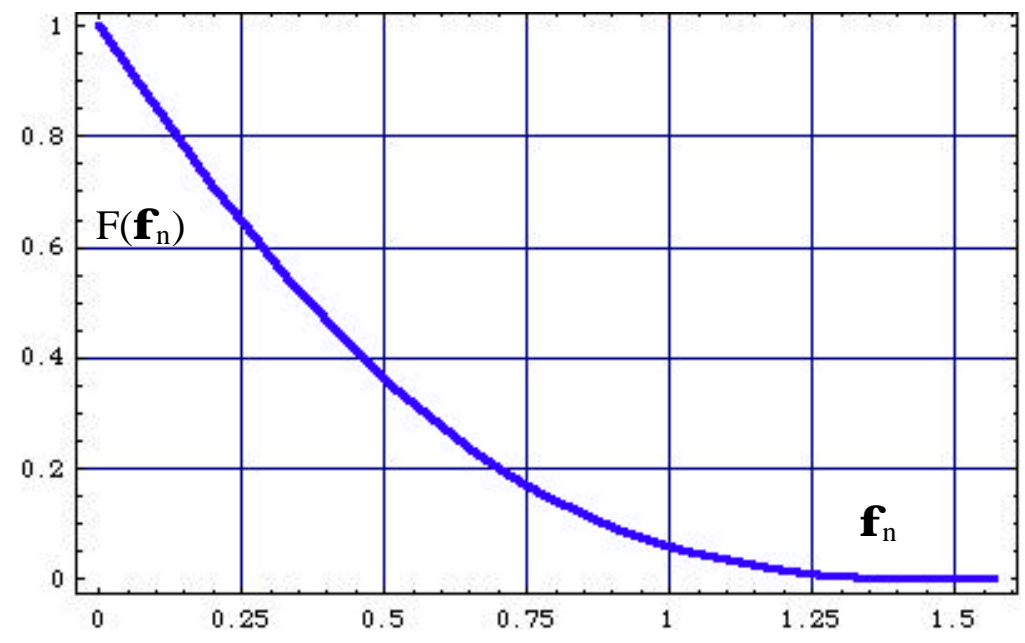

Figure 5. Plot of $F\left(\phi_{n}\right)$ versus $\phi_{n}$ 


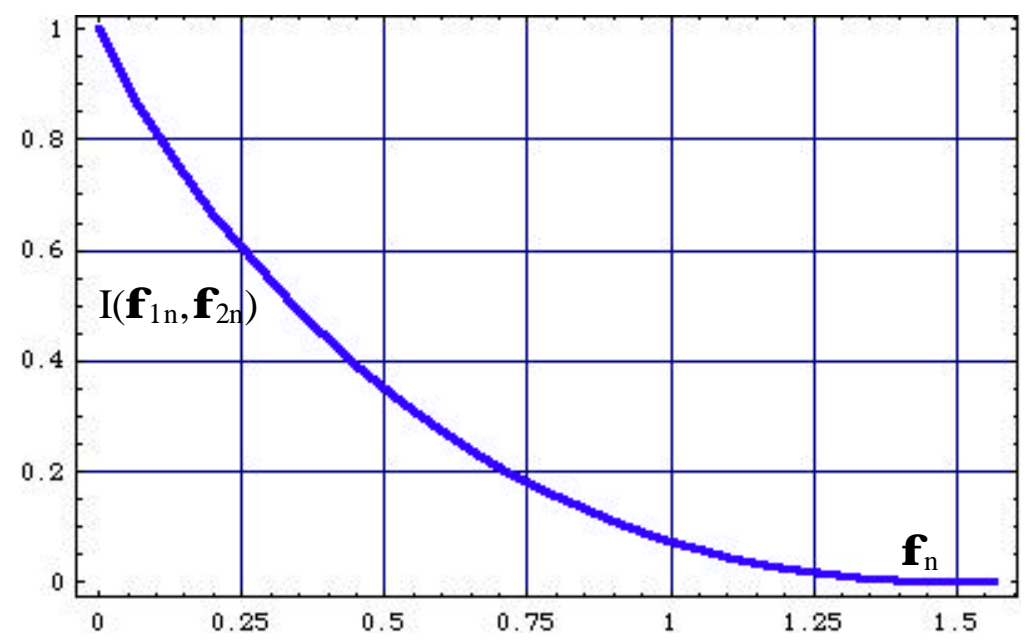

Figure 6. Plot of $\mathrm{I}\left(\phi_{1 \mathrm{n}}, \phi_{2 \mathrm{n}}\right)$ versus RF phase $\phi_{\mathrm{n}}$

In conclusion we have determined that the longitudinal equations of motion for the HNJ method are similar to the conventional ones for constant harmonic acceleration, except that the energy gain per cavity is programmed according to the combination of Eq.s ( 3 and 4). The rate of acceleration cannot be given a-priori, but is determined by the required energy gain program. This is most advantageous indeed in FFAG accelerators where the guiding field does not change with time.

\section{Consequences of the Harmonic Number Jump}

The procedure we have followed applies correctly to the case when beam energy is constantly below the transition energy, namely when $\alpha_{\mathrm{pn}} \gamma_{\mathrm{n}}^{2}<1$. We have indeed set Eq.s (1 to 3) explicitly for this case, that requires a decreasing harmonic number as acceleration proceeds. In the opposite case when the beam energy is above the transition energy, namely that $\alpha_{\mathrm{pn}} \gamma_{\mathrm{n}}^{2}>1$, the harmonic number will increase monotonically. Thus Eq. (2c) is to be replace with $h_{n}-h_{n-1}=+\Delta h$, and a sign minus is to be placed in front of the r.h. side of Eq. (3).

Obviously, to avoid unnecessary beam losses, the number of bunches, that is a constant during acceleration, ought to be always less than the harmonic number at all time. On the other end, because of the change of the revolution period due to the beam velocity variation, the number of RF buckets will vary. The actual distribution of the beam bunches with respect to the available buckets during acceleration is shown in Figure $7 \mathrm{a}$ for the case of acceleration below the transition energy, and in Figure $7 \mathrm{~b}$ for the case above. It is seen that in the former case the beam longitudinal extension at injection ought to be shorter than the revolution period and at most equal to the revolution period at extraction. That is, the number of injected bunches cannot be larger than the RF harmonic number at extraction. The situation is different when the beam is injected at an energy above the transition energy value. In this case the revolution period decreases and the harmonic number increases during acceleration. 


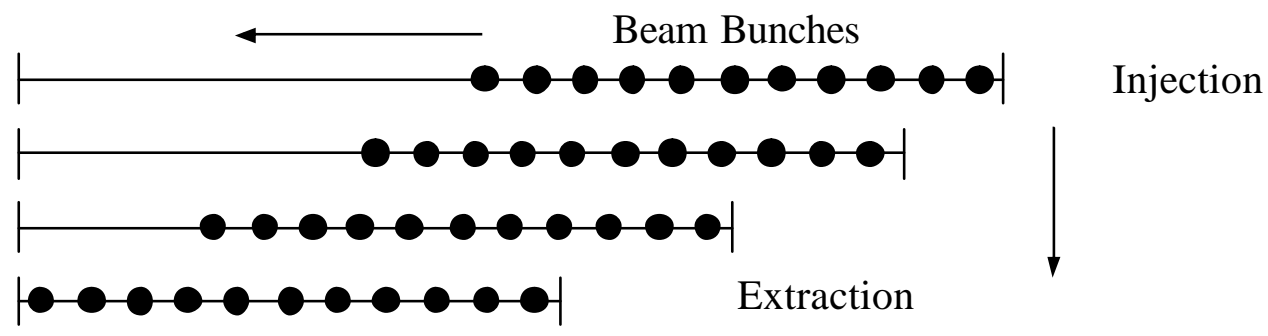

Revolution Period

Figure 7a. Bunch to Bucket Configuration below Transition Energy

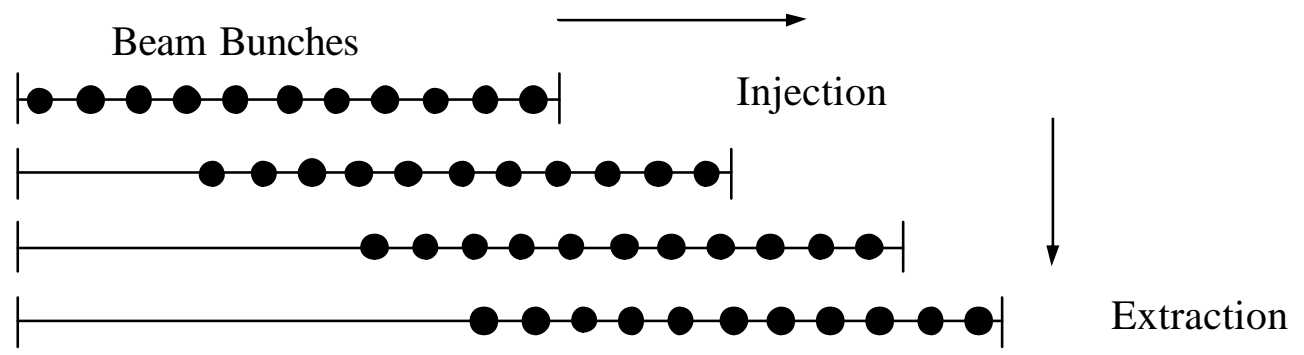

Revolution Period

Figure 7b. Bunch to Bucket Configuration above Transition Energy

\section{Energy Gain Programming}

The acceleration method by HNJ requires that the energy gain at the $\mathrm{n}$-th cavity is given by the combination of Eq.s ( 1 and 3), that is

$$
\mathrm{eV}_{\mathrm{n}} \sin \left(\phi_{\mathrm{n}}\right)=\mathrm{A} \beta_{\mathrm{n}}^{2} \gamma_{\mathrm{n}}^{3} \mathrm{E}_{0} \Delta \mathrm{h} / \mathrm{Q} \mathrm{h}_{\mathrm{n}}\left(1-\alpha_{\mathrm{pn}} \gamma_{\mathrm{n}}^{2}\right)
$$

As an example we show in Figure 8 the energy gain per cavity required for the case of one of the FFAG rings used for Radio-Isotopes Acceleration (RIA) [3]. What is shown is $\mathrm{eV}_{\mathrm{n}} \sin \left(\phi_{\mathrm{n}}\right)$ versus the actual radial position of the beam during acceleration as directly given by Eq. (19) for $\Delta \mathrm{h}=1$. The ring accelerates a beam of ions Uranium +28 from 6 to $50 \mathrm{MeV} / \mathrm{u}$, and has a circumference of $807.091 \mathrm{~m}$. Acceleration occurs at the constant RF frequency of $201.25 \mathrm{MHz}$ with 8 equally-spaced superconducting cavities. Let $\xi_{\text {n }}$ denote the average axial field in the cavity that we take to be made of one single elliptical shape cell with gap g operating in half- wavelength mode. Let also $\lambda$ denote the RF wavelength. The cavity voltage can then be expressed as

$$
\mathrm{V}_{\mathrm{n}}=\mathrm{g} \xi_{\mathrm{n}} \operatorname{TTF}\left(\beta_{0} / \beta\right)
$$

where 
$\mathrm{g}=\lambda \beta_{0} / 2$

and

$\operatorname{TTF}\left(\beta_{0} \beta\right)=\sin \left(\mathrm{p} \beta_{0} / 2 \beta\right) /\left(\mathrm{p} \beta_{0} / 2 \beta\right)$

is the Transit Time Factor (TTF) that is a function of the beam velocity $\beta$, and $\beta_{0}$ is a reference value that corresponds to the gap g for which the TTF has been optimized. Typically $\beta_{0}$ is adjusted to the value of the beam velocity in the middle of the acceleration cycle.

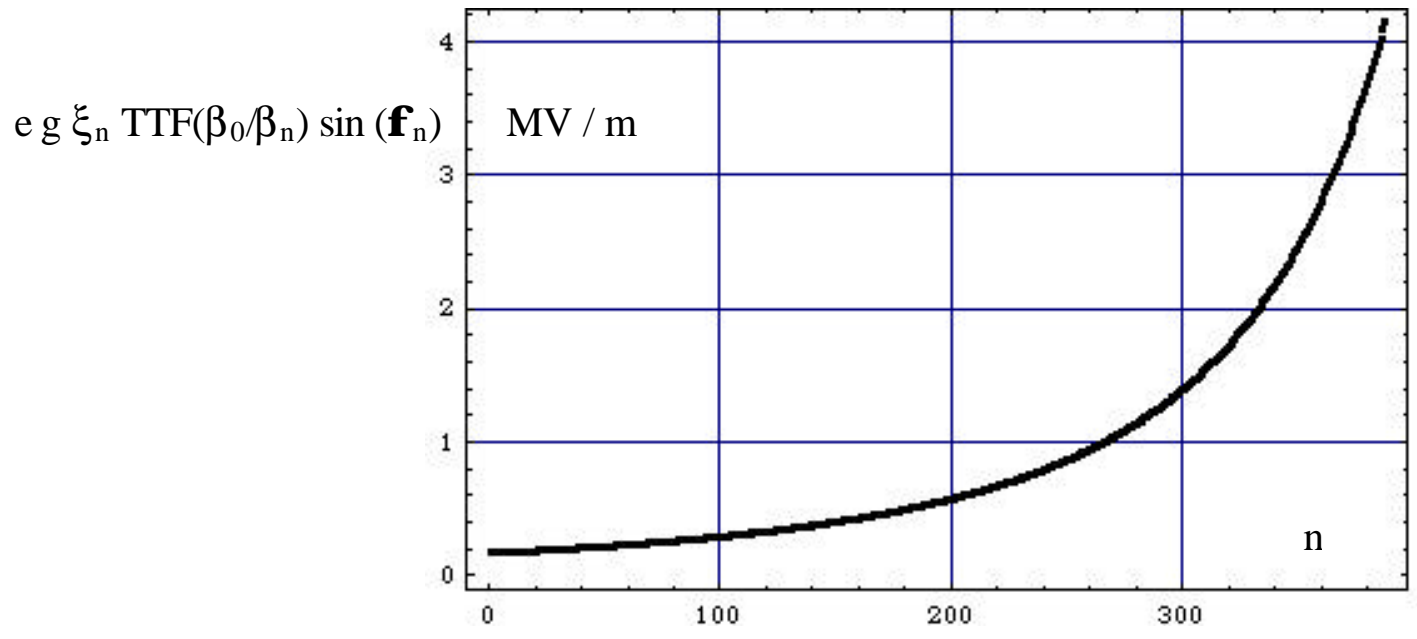

Figure 8. Energy Gain $\Delta \mathbf{E}_{\mathrm{n}}$ Program vs. number of Cavity Crossings

The profile of Figure 8 can be realized in two different modes of operation:

1. Constant RF phase. The average axial accelerating field $\xi_{\mathrm{n}}$ is shown in Figure 9 across the radial aperture for $\phi_{\mathrm{n}}=60^{\circ}$.

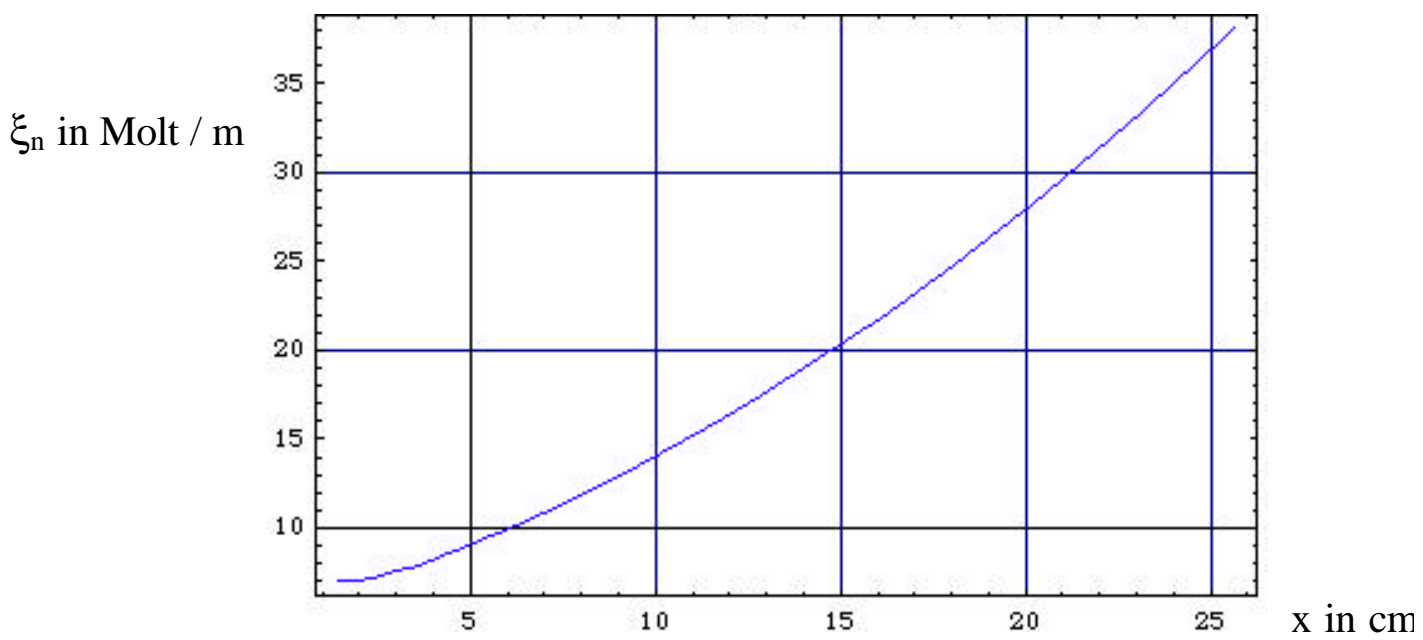

Figure 9. Average Axial Field versus Radial Beam Position with $\phi_{\mathrm{n}}=60^{\circ}$ 
The realization of such field profile across the radial aperture is problematic but not impossible. It could be made with ordinary cavity cells resonating in TM010 mode but displaced horizontally [2].

2. Constant average axial field $\xi_{\mathrm{n}}$. This is obtained by programming the RF phase during the acceleration cycle. The result is shown in Figure 10 that corresponds to the same axial field in all cavities of $38.7 \mathrm{MVolt} / \mathrm{m}$. That includes also the transit time factor TTF. This mode of operation requires that the RF cavities are properly phased with respect to each, and that the phase in all cavities varies from turn to turn In both cases the axial field may be exceedingly too large. It could be lowered with multi-cell cavities, though at the same time the transit time factor behavior may get more irregular due to the large beam velocity change.

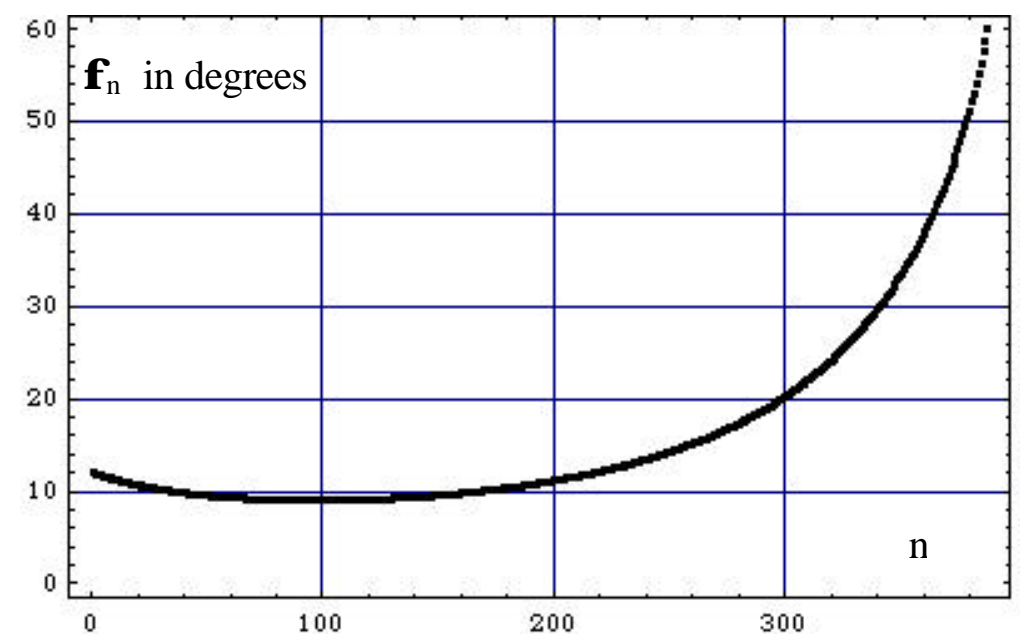

Figure 10. RF Phase $\phi_{\mathrm{n}}$ Program versus no. of Cavity Crossings

\section{Conclusions}

We have investigated a method of low-energy hadron beam acceleration in FFAG accelerators with constant frequency RF superconducting cavities. The method requires an energy gain at each cavity adjusted to allow the beam to skip one or more RF wavelengths between cavities. The energy gain program can be obtained either by requiring a radial profile of the accelerating electric field with the proper design of the cavity, or by setting a constant accelerating field throughout the cycle together to a prescribed program of RF phase. In both approaches transit times factors are evaluated and taken into account as the beam changes velocity over an appreciable range. The analysis performed shows that the longitudinal motion occurs in RF buckets of calculable height and area. 


\section{References}

[1] A. G. Ruggiero, "Design of Proton FFAG Accelerators", Proceedings of FFAG'05 Workshop, Osaka, Japan, Dec. 2005

[2] A. G. Ruggiero, "CW Mode of Operation of a Proton FFAG Accelerator", BNL Internal Report, C-A/AP 218, September 2005.

[3] A. G. Ruggiero, "AGS-less RIA with FFAG Accelerators", BNL Internal Report, C-A/AP 238, May 2006. 